\title{
Raman mapping of a single-layer to double-layer graphene transition
}

\section{Conference Paper}

Author(s):

Graf, Davy; Molitor, Françoise; Ensslin, Klaus (D); Stampfer, Christoph; Jungen, Alain; Hierold, Christofer; Wirtz, Ludger

Publication date:

2007-09

Permanent link:

https://doi.org/10.3929/ethz-b-000003782

Rights / license:

In Copyright - Non-Commercial Use Permitted

Originally published in:

The European Physical Journal Special Topics 148(1), https://doi.org/10.1140/epjst/e2007-00237-1 


\title{
Raman mapping of a single-layer to double-layer graphene transition
}

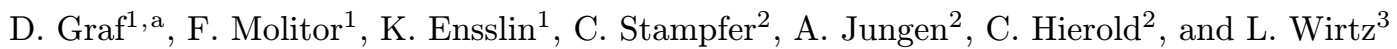 \\ 1 Solid State Physics Laboratory, ETH Zurich, 8093 Zurich, Switzerland \\ 2 Micro and Nanosystems, ETH Zurich, 8092 Zurich, Switzerland \\ 3 Institute for Electronics, Microelectronics, and Nanotechnology (IEMN), CNRS-UMR 8520, \\ BP. 60069, 59652 Villeneuve d'Ascq Cedex, France
}

\begin{abstract}
We report on confocal Raman spectroscopy on a few-layer graphene flake. Adjacent single- and double-layer graphene sections allow mapping the transition in vibrational and electronic properties to a second stacked graphene sheet and with it a weak interlayer coupling. Most prominently the width of the D' peak doubles upon going from a single to a double layer, which can be explained within the double-resonant Raman model. The intensities of the $G$ and G' lines decrease at the crossover to a single layer. Contrary to the $G^{\prime}$ line the $G$ peak position shifts to higher wave numbers, however, not uniformly over the entire section: its frequency fluctuates spatially. The Raman map of the D line intensity shows a non-zero contribution at the boundaries of the flake and the individual sections, which can be attributed either to defects and disorder or to the breakdown of translational symmetry, whereas within the flake no D line signal is detected.
\end{abstract}

\section{Introduction}

Graphite is a highly anisotropic material made of weakly coupled sheets of carbon atoms bonded in a hexagonal lattice. Its building-block, an isolated monoatomic layer called graphene, could only recently be accessed experimentally [1]. Although the stacking of graphene sheets is only supported by a weak interlayer coupling the electronic properties change dramatically for the single-layer graphene as compared to the semi-metallic bulk graphite: the charge carriers are best described as massless Dirac fermions $[2,3]$ with a linear energy dispersion relation. Already for double-layer (also called bilayer) graphene the parabolicity of the valence and conduction bands is recovered at the $\mathrm{K}$ point even though the chirality related to valley pseudo-spin is still preserved. In large magnetic fields this leads to an integer quantum Hall effect different than for few-layer graphene as shown in theory and experiment $[4,5]$.

We present spatially resolved Raman spectra of a graphitic flake composed partly of singleand double-layer graphene allowing to trace directly the change in vibriational and electronic properties at the transition from a single to a double layer. We investigate the intensity of the $\mathrm{D}$ and $\mathrm{G}$ line and discuss the different origin of their shift in peak position. The intensity of the $\mathrm{D}$ line which can be related to defects and disorder is non-zero at the boundary of the flake and at the border between sections of different height, but vanishes within the section [6]. Fluctuations in the $\mathrm{G}$ line shift within a section show, however, that the flake is not homogeneously doped. Surprisingly, the G' line does not shift at all although having a similar intensity decrease from double- to single-layer as the G line. The shape of the D' peak turns out to be the most sensitive parameter to unambiguously discriminate between

\footnotetext{
a e-mail: grafdavy@phys.ethz.ch
} 

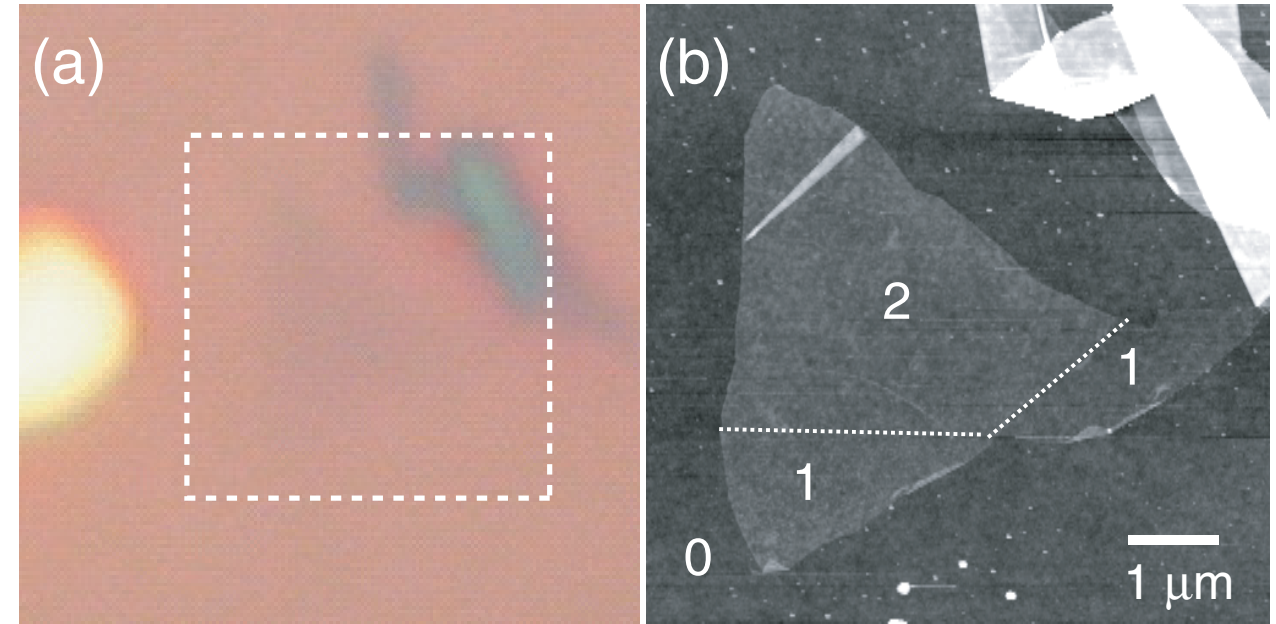

Fig. 1. (a) Optical microscope image of an almost transparent few-layer graphene flake between a metallic marker (left) and a thicker graphite deposition (right) resting on a silicon oxide substrate. (b) SFM micrograph of the few-layer graphene flake (corresponding to the dashed square area in (a)) with a large double-layer and two single-layer sections at the bottom and on the right. The figures indicate the number of layers. Dotted lines mark the boundaries between single- and double-layer graphene sections.

single- and few-layer graphene including double-layer: its width doubles upon the transition from single to double layer since the single Lorentzian peak evolves into a broader structure composed of four peaks $[6,7]$ which can be explained applying double-resonant Raman theory [8].

\section{Sample preparation and experimental setup}

The graphite flakes were prepared by mechanical exfoliation of highly oriented pyrolytic graphite (HOPG) and transfered to a highly doped oxidized silicon waver [1]. With a $300 \mathrm{~nm} \mathrm{SiO}_{2}$ (atomic oxidation process) cap layer the ultrathin graphitic flakes get visible with a standard optical microscope [9]: few-layer graphene flakes are almost transparent (figure 1(a)). Using a scanning force microscope (SFM) we can resolve the contour and the monoatomic step from the singleto double-layer sections shown in figure 1(b). The Raman spectra were acquired using a laser excitation of $532 \mathrm{~nm}(2.33 \mathrm{eV})$ delivered through a single-mode optical fiber, whose spot size is limited by diffraction. Using a long working distance focusing lens with a numerical aperture $\mathrm{NA}=0.80$ we obtain a spot size of about $400 \mathrm{~nm}$ (WITec confocal Raman microscope). With a very low incident power of $4-7 \mu \mathrm{W}$ heating effects can be neglected.

\section{Results and discussion}

\subsection{Intensity of the $D$ and $G$ line}

Two peaks dominate the first-order Raman spectrum of graphite, see figure 2(a): the G line around $1582 \mathrm{~cm}^{-1}$, which can be attributed to the Raman active $\mathrm{E}_{2 g}$ phonon close to the $\Gamma$ point and the $\mathrm{D}$ line around $1350 \mathrm{~cm}^{-1}$ originating from the optical phonon branch next to the $\mathrm{K}$ point. Following a cross section from the double- to the single-layer graphene part ending on the bare silicon substrate (Raman spectra in figure 2(a)) we find that the G peak decreases in amplitude at the transition from double to single layer and shifts at the same time towards higher wave numbers discussed in more details in section 3.3. In the Raman map in figure 2(b) 


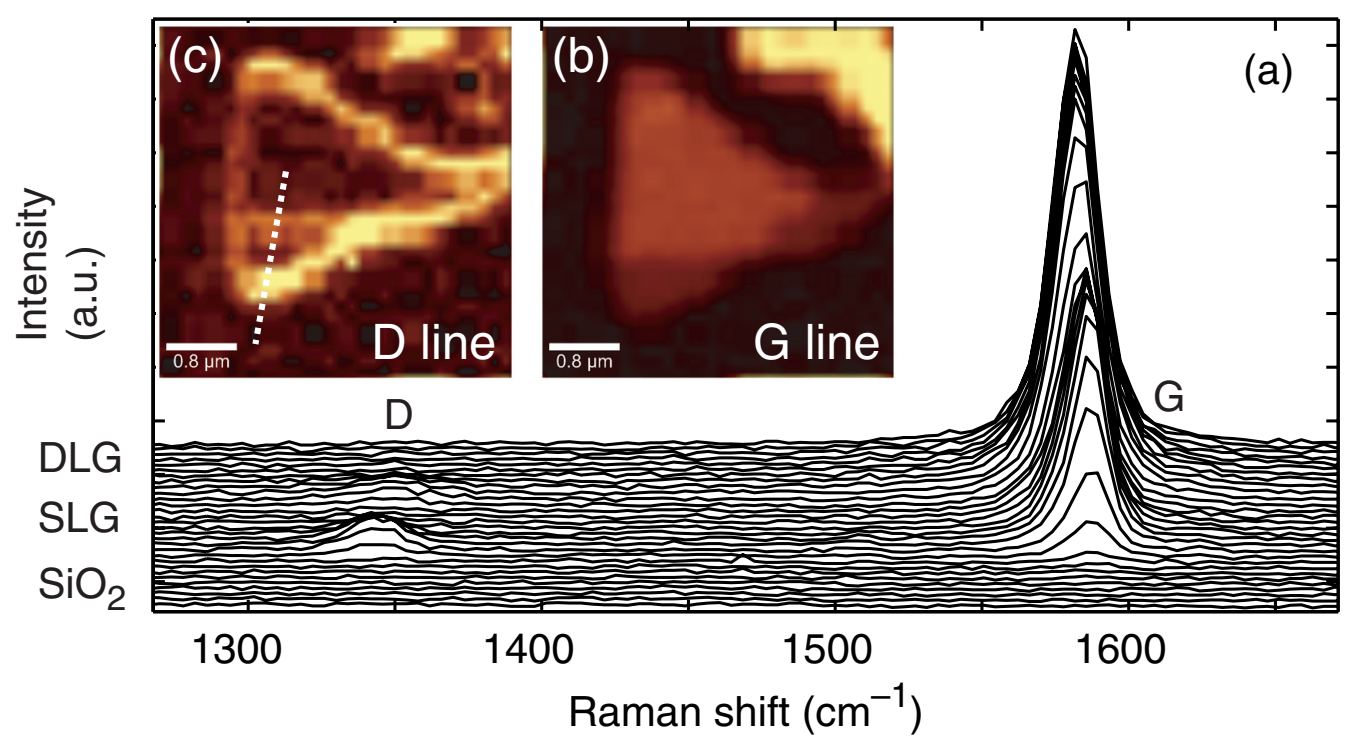

Fig. 2. (a) Raman spectra collected along the white dashed line in (a) showing the D (left) and the G (right) peak. The curves are offset, with the spectra of the bare silicon oxide in the lower and the double-layer graphene section in the upper part. (b) Raman map of the integrated G line intensity $\left(1537-1622 \mathrm{~cm}^{-1}\right)$. (c) Raman map of the integrated D line intensity $\left(1300-1383 \mathrm{~cm}^{-1}\right)$. The color scale varies from dark for zero intensity to bright for large intensities.

the integrated $\mathrm{G}$ line intensity is reduced for the lower and the right part of the flake which can clearly be assigned to the two single-layer graphene sections labelled in figure 1(b). The D peak in the set of curves plotted in figure 2(a) is non-zero at the boundary of the flake and at the step from double- to single-layer graphene. At the cross over from single-layer graphene to the silicon oxide substrate the peak is shifted to smaller wave numbers discussed in more details in section 3.2. Figure 2(c) confirms this spatial D line intensity distribution: the integrated signal of the $\mathrm{D}$ line follows the outer boundary of the flake and also the borderlines between sections of different height [6]. Earlier experiments showed that the appearance of the D line can be associated with the occurrance of local defects and also disorder in general [10-12]. We therefore conclude that the inner part of the flake is free of defects and disorder. The appearance of a measurable $\mathrm{D}$ peak amplitude at the boundaries can, however, also be assigned to the breakdown of translational symmetry [10].

\subsection{Peak width of the $D^{\prime}$ line}

Some peculiarities of the D line could be explained within the double-resonant Raman model developed by Thomsen and Reich [8], e.g. the dispersion with the wave length of the incident laser light [11]. The finite wave vector of the phonon transition makes scattering process of the resonantly excited electron dependent on the electronic band structure. For the D line an elastic scattering process restores momentum conservation, which explains its sensitivity to defects and disorder. In the case of the D' line constituting the related second order process a second phonon with opposite wave vector will provide the backscattering mechanism. The lifting of the band degeneracy for double-layer graphene due to the interplane interaction allows for four possible phonons in contrast to the single scattering process in the case of the conelike electronic band structure of single-layer graphene. We expect therefore a broadening of the D' peak at the transition from single- to double-layer graphene from a single peak to a convolution of four peaks, which is verified in the experimental data depicted in figure 3(a) $[6,7]$. In addition, a downshift of the single-layer peak compared to the spectral center of mass of the double-layer peak can be observed, which is consistent with the downshift of the D line 

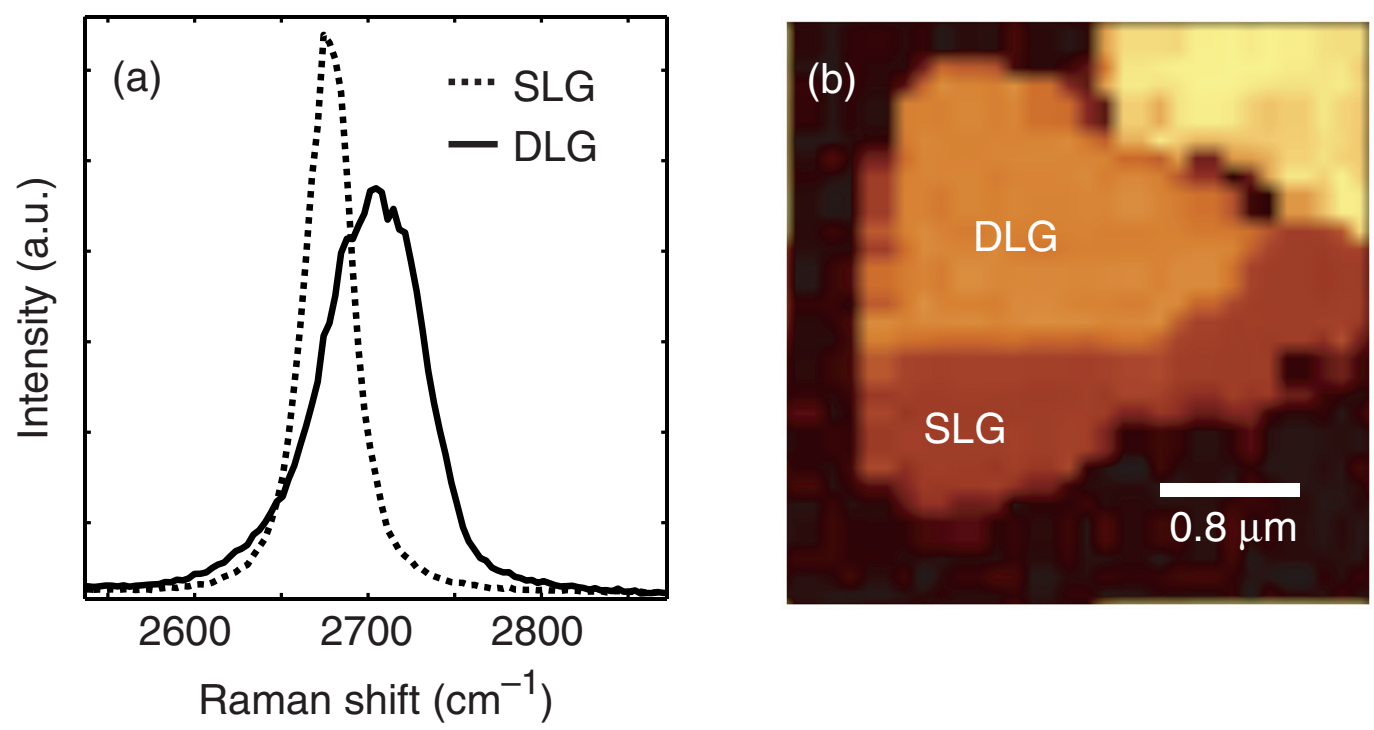

Fig. 3. (a) Raman spectra around the D' line for the single-layer graphene (SLG, dotted curve) and the double-layer graphene (DLG, solid curve). (b) Raman map of the FWHM of the D' line. Color scale: The brighter the larger the width.

peak for pure single-layer contribution at the step from single-layer graphene to the silicon oxide substrate. The Raman map of the full width at half maximum (FWHM) of the D' line provides a clear contrast between single- and double-layer graphene. For three- and four layers the integrated G peak intensity increases linearly while the width of the D' line stays almost constant such that can be used to discriminate single- from double and few-layer graphene [6].

\subsection{Peak position of the $G$ and the G' line}

At the crossover from single- to double-layer graphene the position of the G peak shifts towards higher wave numbers. The magnitude of the shift is not a constant but varies for different flakes [6]. For that it is important to investigate the spatial variation of that shift within the same flake. In figures 4(a) and (b) the peak position is shown in two different color scales: in (a) the single-layer sections are highlighted whereas in (b) the contrast is optimized for the double-layer section. In both cases fluctuations reveal that the shift is not a property of the entire flake or section but varies in space. A recently published experiment ascribes the shift of the G peak frequency to the position of the Fermi level i.e. the amount of doping [13]. The charge transfer is mainly due to adsorbed molecules [14], such that the sensitivity to donors on the surface is largest for single- and double-layer graphene (considering the very short screening length of the order of the interplane distance). We suggest that the spatial inhomogeneity of the absorbed molecules is reflected in the fluctuations of the $\mathrm{G}$ peak position displayed in figures $4(\mathrm{a})$ and (b). The G line shift is thus complementary to the D line intensity in characterizing the electronic properties of few-layer graphene.

The counterpart of the G line in the second order Raman spectrum, the G' line around $3248 \mathrm{~cm}^{-1}$, is ten times weaker in integrated intensity as seen in the cross sectional plot in figure 5(a) and decreases when reducing the number of layers from two to one, even tough not with clear plateaus as for the integrated G line intensity. Contrary to the G line that shifts by $4 \mathrm{~cm}^{-1}$ at the transition from the double to the single layer, the $\mathrm{G}$ ' line does not shift noticeably (figure 5(b)). This can be understood as follows: The G line is due to excitation (or absorption) of a LO phonon at $\Gamma$, i.e. for vanishing phonon wave vector $q$. The strong shift of its frequency upon doping is a consequence of the Kohn-Anomaly of this mode for $q=0$ [15]. According to the double-resonant Raman model [8], the G' line - which has more than twice the frequency 

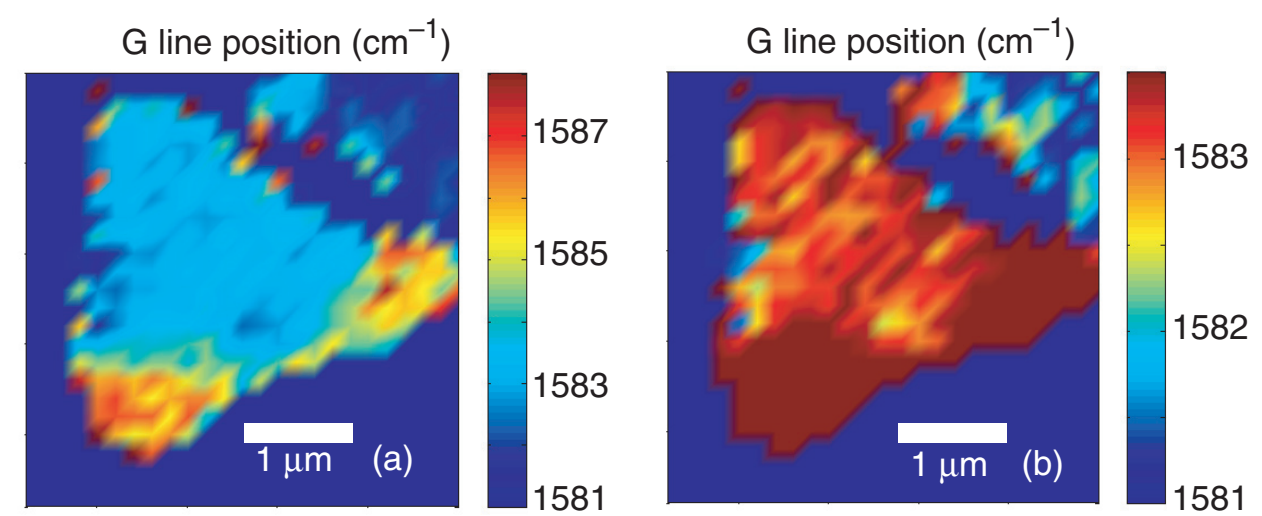

Fig. 4. Raman map of the G line position: Contrast optimized for (a) the single-layer graphene sections and (b) for the double-layer graphene section.
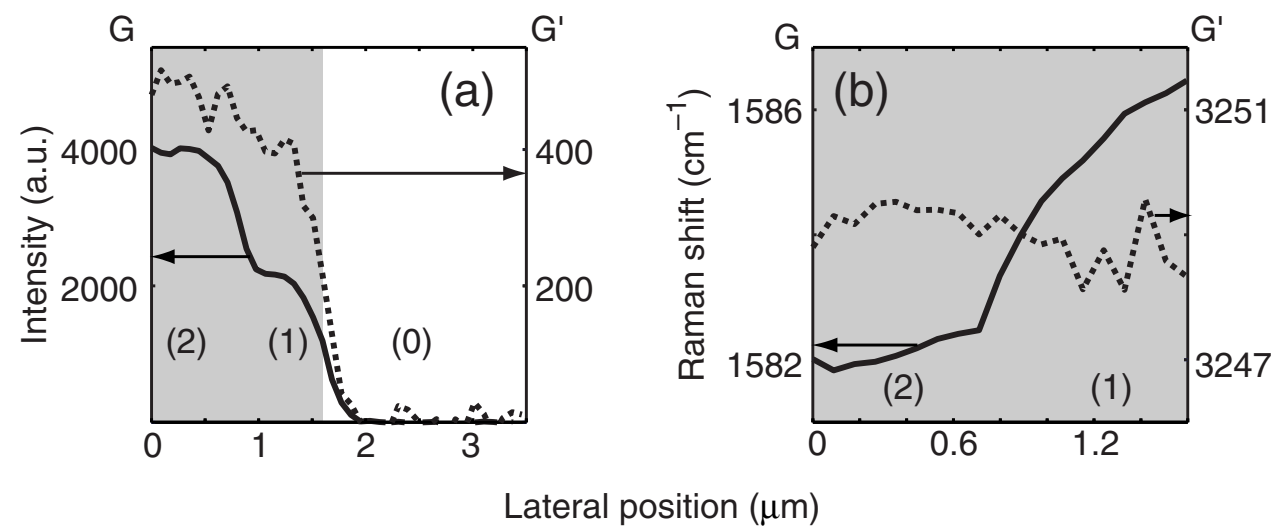

Fig. 5. (a) Raman intensity cross section along the white dashed line in figure 2(a) showing the integrated intensity of the $G$ line (solid, left scale) and the G' line (dotted, right scale). (b) Raman line position of the G peak (solid, left scale) and G' peak (dashed, right) for the part in (a) with the gray background. The figures in brackets indicate the number of layers.

of the G line - is due to the excitation (or absorption) of two LO-phonons with $q \neq 0$. At $q \neq 0$, the LO-phonon frequency is less affected by doping. Therefore the position of the G' line hardly moves at the transition from single to double layer.

\section{Conclusion}

Raman spectroscopy turns out to be a powerful tool to investigate the transition from singleto double-layer graphene. Collecting spatially resolved Raman spectra of a graphitic flake with neighboring single- and double-layer sections allows us to compare the contribution of the various phonon modes in absolute values. The $\mathrm{G}$ and $\mathrm{G}$ ' line intensities decrease with the number of layers. The $\mathrm{G}$ peak position shifts to higher wave numbers for the single-layer graphene while the G' peak position keeps constant. The shift of the G peak position is, however, not constant in space but fluctuates within a given section. The width of the D' peak is highly sensitive to the crossover from single- to double- and few-layer graphene, which is explained by a peak splitting following the double-resonant Raman model. The mapping of the integrated D line intensity indicates that disorder and defects as well as translational symmetry breaking are mostly located at the steps and edges of the graphite flake. 
We acknowledge stimulating discussions with A.H. Castro Neto. Financial support from the Swiss Science Foundation (Schweizerischer Nationalfonds) is gratefully acknowledged.

\section{References}

1. K.S. Novoselov, A.K. Geim, S.V. Morozov, D. Jiang, Y. Zhang, S.V. Dubonos, I.V. Grigorieva, A.A. Firsov, Science 306, 666 (2004)

2. K.S. Novoselov, A.K. Geim, S.V. Morozov, D. Jiang, M.I. Katsnelson, I.V. Grigorieva, S.V. Dubonos, A.A. Firsov, Nature 438, 197 (2005)

3. Y. Zhang, Y.-W. Tan, H.L. Stormer, P. Kim, Nature 438, 201 (2005)

4. E. McCann, V.I. Fal'ko, Phys. Rev. Lett. 96, 086805 (2006)

5. K.S. Novoselov, E. McCann, S.V. Morozov, V.I. Fal'ko, M.I. Katsnelson, U. Zeitler, D. Jiang, F. Schedin, A.K. Geim, Nat.Phys. 2, 177 (2006)

6. D. Graf, F. Molitor, K. Ensslin, C. Stampfer, A. Jungen, C. Hierold, L. Wirtz, Nano Lett., cond-mat/0607562 (2006) (in press)

7. A.C. Ferrari, J.C. Meyer, V. Scardaci, C. Casiraghi, M. Lazzeri, F. Mauri, S. Piscanec, D. Jiang, K.S. Novoselov, S. Roth, A.K. Geim, Phys. Rev. Lett. 97, 187401 (2006)

8. C. Thomsen, S. Reich, Phys. Rev. Lett. 85, 5214 (2000)

9. K.S. Novoselov, D. Jiang, F. Schedin, T.J. Booth, V.V. Khotkevich, S.V. Morozov, A.K. Geim, PNAS 102, 10451 (2005)

10. F. Tuinstra, J.L. Koenig, J. Chem. Phys. 53, 1126 (1970)

11. R.P. Vidano, D.B. Fischbach, L.J. Willis, T.M. Loehr, Solid State Commun. 39, 341 (1981)

12. Y. Wang, D.C. Alsmeyer, R.L. McCreery, Chem. Mater. 2, 557 (1990)

13. S. Pisana, M. Lazzeri, C. Casiraghi, K.S. Novoselov, A.K. Geim, A.C. Ferrari, F. Mauri, cond-mat/0611714 (2006)

14. F. Schedin, K.S. Novoselov, S.V. Morozov, D. Jiang, E.H. Hill, P. Blake, A.K. Geim, cond-mat/0610809 (2006)

15. M. Lazzeri, F. Mauri, Phys. Rev. Lett., cond-mat/0611708 (to appear) 